\title{
FACTORIZATIONS OF FINITE GROUPS BY CONJUGATE SUBGROUPS WHICH ARE SOLVABLE OR NILPOTENT
}

\author{
MARTINO GARONZI, DAN LEVY, ATTILA MARÓTI, AND IULIAN I. SIMION
}

\begin{abstract}
We consider factorizations of a finite group $G$ into conjugate subgroups, $G=A^{x_{1}} \cdots A^{x_{k}}$ for $A \leq G$ and $x_{1}, \ldots, x_{k} \in G$, where $A$ is nilpotent or solvable. We derive an upper bound on the minimal length of a solvable conjugate factorization of a general finite group which, for a large class of groups, is linear in the non-solvable length of $G$. We also show that every solvable group $G$ is a product of at most $1+c \log |G: C|$ conjugates of a Carter subgroup $C$ of $G$, where $c$ is a positive real constant. Finally, using these results we obtain an upper bound on the minimal length of a nilpotent conjugate factorization of a general finite group.
\end{abstract}

\section{INTRODUCTION}

During the last few years there appeared several papers which consider the possibilities to express a finite group $G^{1}$ as a product of conjugates of a proper subgroup (e.g., $[3,8,16,17,23-26,28,29]$ ). In particular, in [8] it is proved that every non-solvable group can be written as a product of three conjugates of at least one of its proper subgroups. A key step in the proof shows that almost simple groups with socle of Lie type (which, in an appropriate sense, constitute the bulk of the almost simple groups) can be written as a product of three conjugates of a proper solvable subgroup. In [17] it is shown that every simple group of Lie type can be written as a product of four nilpotent subgroups. These results naturally raise the question which we address in the present paper: For a group $G$, what can be said about the minimal number $m$ such that $G$ is the product of $m$ conjugates of $A \leq G$ where $A$ is known to be solvable or to be nilpotent?

Let us formalize our main notions. For a group $G$ and $A \leq G$, a conjugate product factorization (a cp-factorization) of length $k$ of $G$ by $A$ is a factorization $G=A_{1} \cdots A_{k}$ where $A_{1}, \ldots, A_{k}$ are all conjugate to $A$ and the product is the setwise product. Denoting the normal closure of $A$ in $G$ by $A^{G}$, an elementary argument shows that $A^{G}$ is equal to a product of conjugates of $A$. Thus, a necessary and

Date: February 11, 2016.

2000 Mathematics Subject Classification. 20B15, 20B40, 20 D05.

Key words and phrases. products of conjugate subgroups, non-solvable length, Carter subgroup.

M.G. acknowledges the support of CAPES and the University of Brasilia (UnB).

A.M. was supported by an Alexander von Humboldt Fellowship for Experienced Researchers, by MTA Rényi "Lendület" Groups and Graphs Research Group, and by OTKA grants K84233 and K115799.

I.S. acknowledges the support of the University of Padova (grants CPDR131579/13 and CPDA125818/12).

${ }^{1}$ All groups considered are assumed to be finite. 
sufficient condition for the existence of the factorizations we are interested in is $G=A^{G}$.

Definition 1.1. Let $G$ be a group and $A \leq G$. Then $\gamma_{\mathrm{cp}}^{A}(G)$ is the smallest number $k$ such that $G$ equals a product $A_{1} \cdots A_{k}$ of conjugates of $A$ or $\infty$ if no such $k$ exists. We also set

$$
\gamma_{\mathrm{cp}}(G):=\min \left\{\gamma_{\mathrm{cp}}^{A}(G) \mid A<G\right\},
$$

where $\min \{\infty\}:=\infty$.

It is easy to see that if $A<G$ and $G$ is nilpotent then $\gamma_{\mathrm{cp}}^{A}(G)=\infty$, and that in general $\gamma_{\mathrm{cp}}^{A}(G) \geq 3$. As mentioned above, if $G$ is non-solvable then $\gamma_{\mathrm{cp}}(G)=3$ ([8]). In contrast, for $G$ solvable non-nilpotent, $\gamma_{\mathrm{cp}}(G)$ is not bounded above by a universal constant, but we do have $\gamma_{\mathrm{cp}}(G) \leq 4 \log _{2}|G|[16 \text {, Theorems } 4 \text { and } 5]^{2}$.

The aim of the present paper is to obtain upper bounds on the following two quantities:

$$
\gamma_{\mathrm{cp}}^{\mathrm{s}}(G):=\min \left\{\gamma_{\mathrm{cp}}^{A}(G) \mid A \leq G \text { is solvable }\right\},
$$

and

$$
\gamma_{\mathrm{cp}}^{\mathrm{n}}(G):=\min \left\{\gamma_{\mathrm{cp}}^{A}(G) \mid A \leq G \text { is nilpotent }\right\} .
$$

Observe that $\gamma_{\mathrm{cp}}^{\mathrm{s}}(G)=1\left(\gamma_{\mathrm{cp}}^{\mathrm{n}}(G)=1\right)$ if and only if $G$ is solvable (nilpotent). In general, both of these quantities are natural numbers. For if $G$ is nilpotent or if $G$ contains a proper subgroup which is both nilpotent and non-normal maximal, the claim is clear. Otherwise $G=A_{1} \cdots A_{k}$ is a cp-factorization where $A_{1}$ is some maximal non-normal subgroup of $G$, and by induction $A_{1}$ has a nilpotent cp-factorization.

Our upper bound on $\gamma_{\mathrm{cp}}^{\mathrm{s}}(G)$ is expressed in terms of the non-solvable length of the group $G$ which is defined as follows (we denote by $R(G)$ the solvable radical of $G$ and by $\operatorname{soc}(G)$ the socle of $G)$.

Definition 1.2. Let $G$ be a group. The non-abelian socle series of $G$ is the unique normal series $R(G)=H_{1} \leq \ldots \leq H_{t}=G$ of $G$ which satisfies the following conditions:

(i) for all $1 \leq i \leq(t-1) / 2$ we have $H_{2 i+1} / H_{2 i}=R\left(G / H_{2 i}\right)$,

(ii) for all $1 \leq i \leq t / 2$ we have $H_{2 i} / H_{2 i-1}=\operatorname{soc}\left(G / H_{2 i-1}\right)$.

The number $\lfloor t / 2\rfloor$ is called the non-solvable length of $G$ and will be denoted $\lambda(G)$.

Theorem 1.3. Let $G$ be a group. Then $\gamma_{\mathrm{cp}}^{\mathrm{s}}(G) \leq 1+c(G) \lambda(G)$, where $c(G)=$ $\max \left\{36,12 \log _{2} n\right\}$ and $n$ is the largest integer such that $G$ has a composition factor $\operatorname{Alt}(n)$ ( $n=2$ if no such factor exists).

The non-abelian socle series has important applications in advanced computations in finite groups [19, Chapter 10]. Furthermore, the parameter $\lambda(G)$ is the subject of a recent interesting paper by Khukhro and Shumyatsky [14], whose main result is the upper bound $\lambda(G) \leq 2 L_{2}+1$, where $L_{2}$ is the maximum 2-length of a solvable subgroup of $G$. The 2-length of a solvable group $H$ is the minimal number of 2-factors that can occur in a normal series $\left\{1_{H}\right\}=H_{1} \leq \ldots \leq H_{l}=H$ such that for each $0 \leq i \leq l-1$ each factor $H_{i+1} / H_{i}$ is either a 2-group or an odd order group. Here we prove another upper bound on $\lambda(G)$ which we later employ.

\footnotetext{
${ }^{2}$ In fact, this bound is somewhat improved by Theorem 1.6 below.
} 
Theorem 1.4. Let $G$ be a non-solvable group, and let $k$ be the number of nonabelian composition factors appearing in a composition series of $G$ (repetitions counted). Then $\lambda(G) \leq \log _{5}(4 k+1)$.

Remark 1.5. Observe that for any positive integer $m$, the $m$-fold iterated wreath product $W_{m}$, where $W_{1}:=$ Alt (5) and for each $2 \leq i \leq m, W_{i}:=W_{i-1}$ wr Alt (5), attains the upper bound of Theorem 1.4. One checks, using the definition of $W_{m}$ (view $k$ as a function of $m$ ), that $\lambda\left(W_{m}\right)=m$, and that $k(m)=\frac{5^{m}-1}{4}$. Therefore $\lambda\left(W_{m}\right)=\log _{5}(4 k(m)+1)$.

We also remark in passing, that Theorem 1.4 can be generalized to get an upper bound on the non-p-solvable length of a group, introduced by Khukhro and Shumyatsky in [14].

Next we obtain an upper bound on $\gamma_{\mathrm{cp}}^{\mathrm{n}}(G)$ for solvable groups $G$. Recall that a Carter subgroup of a group $G$ is a self-normalizing nilpotent subgroup (see [9]). If $G$ is solvable then it has a unique conjugacy class of Carter subgroups.

Theorem 1.6. Any solvable group $G$ is a product of at most $1+c_{\mathrm{ca}} \log _{2}|G: C|$ conjugates of a Carter subgroup $C$, where $0<c_{\mathrm{ca}} \leq 3 / \log _{2}(5)<1.3$ is a universal constant.

Finally, combining the last theorem with the previous bounds on $\gamma_{\mathrm{cp}}^{\mathrm{s}}(G)$ and on $\lambda(G)$ we will prove:

Theorem 1.7. For any non-nilpotent group $G$ there exists a nilpotent $A<G$ such that

$$
\gamma_{\mathrm{cp}}^{\mathrm{n}}(G) \leq \gamma_{\mathrm{cp}}^{A}(G) \leq 1+c_{u s}\left(\log _{2}|G: A|\right)\left(\log _{2} \log _{2}|G: A|\right)^{2}
$$

where $c_{u s}$ is a universal constant.

\section{Solvable cp-Factorizations}

In this section we prove Theorem 1.3. The proof is based on reducing the problem of finding an upper bound on $\gamma_{\mathrm{cp}}^{\mathrm{s}}(G)$ for a general group $G$ to finding an upper bound on the minimal length of a special kind of a solvable conjugate factorization of a simple non-abelian group.

Definition 2.1. A cp-factorization $G=A_{1} \cdots A_{k}$ of a group $G$ by $A \leq G$ will be called a special solvable cp-factorization if the following conditions hold:

(i) $A$ is solvable.

(ii) $A$ is self-normalizing in $G$.

(iii) For any $\alpha \in A u t(G)$ there exists $g \in G$ such that $A^{\alpha}=A^{g}$.

We prove that special solvable cp-factorizations exist for any group $G$.

Lemma 2.2. Let $G$ be a group, $p$ a prime, and let $P$ be a Sylow p-subgroup of $G$. Then $A:=N_{G}(P)$ satisfies properties (ii) and (iii) in Definition 2.1, and $G$ is a product of some conjugates of $A$ in $G$. If, in addition, $A$ is solvable then this product is a special solvable conjugate factorization of $G$. Furthermore, if $p=2$ then $A$ is solvable so any non-nilpotent group $G$ has at least one special solvable conjugate factorization.

Proof. It is well-known that as a consequence of Sylow's theorems, properties (ii) and (iii) in Definition 2.3 are satisfied by any Sylow normalizer subgroup of $G$ (see, for instance, $[27,5.13,5.14])$. In order to show that $G$ is a product of conjugates of 
$A$ it suffices to prove that $G=A^{G}$. Observe that $P$ is a Sylow $p$-subgroup of $A^{G}$ and clearly $A^{G} \unlhd G$. Hence, by Frattini's argument, $G=A\left(A^{G}\right)=A^{G}$. Finally, if $p=2$ then $A$ is solvable by the Odd Order Theorem.

Definition 2.3. For a group $G$ we denote by $\gamma_{\mathrm{cp}}^{\mathrm{ss}}(G)$ the minimal length of a special solvable cp-factorization of $G$, if such a factorization exists, or $\gamma_{\mathrm{cp}}^{\mathrm{ss}}(G)=\infty$ otherwise. For a prime $p$ we let $\gamma_{\mathrm{cp}}^{p}(G)$ denote the minimal length of a cp-factorization of $G$ whose factors are conjugates of a solvable normalizer in $G$ of a Sylow p-subgroup of $G$, if such a factorization exists, or $\gamma_{\mathrm{cp}}^{p}(G)=\infty$ otherwise.

By Lemma 2.2, $\gamma_{\mathrm{cp}}^{\mathrm{ss}}(G) \leq \gamma_{\mathrm{cp}}^{p}(G)$ holds for every prime $p$.

2.1. Special solvable factorizations of simple groups. Here we obtain an upper bound on $\gamma_{\mathrm{cp}}^{\mathrm{ss}}(S)$, where $S$ is a simple non-abelian group. We discuss separately simple groups of Lie type, alternating groups and simple sporadic groups together with the Tits group.

Lemma 2.4. If $S$ is a simple group of Lie type of characteristic $p$ then $\gamma_{\mathrm{cp}}^{\mathrm{ss}}(S)=$ $\gamma_{\mathrm{cp}}^{p}(S)=3$.

Proof. $S$ is a group with a split $B N$-pair, where $B$, the Borel subgroup of $S$, is solvable and is the normalizer of a Sylow $p$-subgroup of $S$. Hence $\gamma_{\mathrm{cp}}^{p}(S)=3$ by [8, Theorem 3].

Lemma 2.5. If $S \cong$ Alt $(n)$ for $n \geq 5$ then $\gamma_{\mathrm{cp}}^{\mathrm{ss}}(S)<12 \log _{2}(n)$.

Proof. We first show that for all $n \geq 2$ the symmetric group $S_{n}$ is a product of less than $4 \log _{2}(n)$ Sylow 2-subgroups, adjusting the ideas of the proof of [1, Theorem $2]$ to our needs. For any positive integer $n$ set $\Omega_{n}:=\{1,2, \ldots, n\}$. Denote by $f(n)$ the minimal length of a cp-factorization of $G$ whose factors are Sylow 2-subgroups. First we show that $f(n+1) \leq f(n)+2$. Let $A \cong S_{n}$ be the point stabilizer of 1 , with respect to the natural action of $S_{n+1}$ on $\Omega_{n+1}$. Then $A$ is a product of $f(n)$ Sylow 2-subgroups of $A$ each of which is a subgroup of a Sylow 2-subgroup of $S_{n+1}$. Next we prove that there exist two Sylow 2-subgroups $P$ and $Q$ of $S_{n+1}$, such that $P Q$ contains elements $g_{1}=1_{S_{n+1}}, g_{2}, \ldots, g_{n+1}$ satisfying $(1) g_{i}=i$ for each $1 \leq i \leq n+1$ ((1) $g_{i}$ stands for the image of $1 \in \Omega_{n+1}$ under the action of $\left.g_{i} \in S_{n+1}\right)$. Note that a subset $\left\{g_{1}, \ldots, g_{n+1}\right\}$ of $S_{n+1}$ whose elements satisfy the last condition is a right transversal of $A$ in $S_{n+1}$, for if $i \neq j$ then (1) $g_{i} g_{j}^{-1} \neq 1$, implying $g_{i} g_{j}^{-1} \notin A$. Clearly, if $P Q$ contains a right transversal of $A$ in $S_{n+1}$, we have $A P Q=S_{n+1}$, and $f(n+1) \leq f(n)+2$ follows.

Let $k$ be the unique integer satisfying $2^{k} \leq n+1<2^{k+1}$. We can choose $P$ to be a Sylow 2-subgroup of $S_{n+1}$ containing $\left\langle\left(1, \ldots, 2^{k}\right)\right\rangle$, and $Q$ a Sylow 2subgroup of $S_{n+1}$ containing $\left\langle\left(n-2^{k}+2, \ldots, n+1\right)\right\rangle$. These two cyclic subgroups act transitively on their supports, and their supports have at least one point in common. Hence $P Q$ contains a subset $\left\{g_{1}, \ldots, g_{n+1}\right\}$ having the desired property.

Next we show that if $n$ is even then $f(n) \leq 2 f(2)+f(n / 2)$. In this case, $\Omega_{n}$ is in bijection with the set $\widetilde{\Omega}_{n}:=\{1,2\} \times \Omega_{n / 2}$. The natural action of $S_{n}$ on $\Omega_{n}$ induces an action of $S_{n}$ on $\widetilde{\Omega}_{n}$. Let $A$ be the subgroup consisting of all $g \in S_{n}$ such that for any $(a, b) \in \widetilde{\Omega}_{n}$ we have $(a, b) g=(a, x)$ for some $x \in \Omega_{n / 2}$ and similarly, $B$ is the subgroup of $S_{n}$ preserving the second coordinate of the $\widetilde{\Omega}_{n}$ 
element. Then, $A \cong\left(S_{n / 2}\right)^{2}$ and $B \cong\left(S_{2}\right)^{n / 2}$. By [1, Lemma 4], $S_{n}=B A B$. This gives $f(n) \leq 2 f(2)+f(n / 2)$.

Using these two inequalities we prove $f(n)<4 \log _{2}(n)$ by induction. The base case is $n=2$ and for it we have $f(2)=1<4$. Suppose $n>2$. If $n$ is even then

$$
f(n) \leq 2+f(n / 2)<2+4 \log _{2}(n / 2)<4 \log _{2}(n) .
$$

If $n$ is odd, then

$$
f(n) \leq 2+f(n-1) \leq 4+f((n-1) / 2)<4+4 \log _{2}((n-1) / 2)<4 \log _{2}(n) .
$$

Next we show that for $n \geq 6$ the group $\operatorname{Alt}(n)$ is a product of at most $12 \log _{2}(n)$ Sylow 2-subgroups. The group Alt $(n)$ acts transitively on $P_{2}\left(\Omega_{n}\right)$ the set of all $n(n-1) / 2$ subsets of $\Omega_{n}$ of size 2 . One can check that the stabilizer of a subset of size 2 of $\Omega_{n}$ is isomorphic to $S_{n-2}$. Let $H_{1}$ and $H_{2}$ be the stabilizers of $\{1,2\}$ and $\{n-1, n\}$ respectively. We claim that $\operatorname{Alt}(n)=H_{1} H_{2} H_{1}$. Notice that this claim together with our previous claim that $S_{n}$ is a product of less than $4 \log _{2}(n)$ Sylow 2-subgroups, finish the proof. We have $H_{2}=H_{1}^{g}$ with $g=(1, n-1)(2, n)$. By $[8$, Theorem 1 part 2 (ii)] it is sufficient to show that $\{1,2\} H_{2}$ intersects every $H_{1}$ orbit $O$ on $P_{2}\left(\Omega_{n}\right)$. Let $\{i, j\} \in O$ be arbitrary. If $\{i, j\} \cap\{n-1, n\}=\emptyset$, then there exists an $h \in H_{2}$ such that $\{1,2\} h=\{i, j\}$. On the other hand, if $\{i, j\} \cap\{n-1, n\} \neq \emptyset$ then, since $n \geq 6$, there exists $h_{1} \in H_{1}$ so that $\{i, j\} h_{1} \cap\{n-1, n\}=\emptyset$, and so, since $\{i, j\} h_{1} \in O$ we reduce to the previous case.

Finally, $\gamma_{\mathrm{cp}}^{\mathrm{ss}}(\operatorname{Alt}(5))=3$ by Lemma 2.4 since $\operatorname{Alt}(5) \cong P S L(2,4)$ is of Lie type in characteristic 2. For $n \geq 6$ we have shown that Alt $(n)$ is a product of at most $12 \log _{2}(n)$ Sylow 2-subgroups, hence $\gamma_{\mathrm{cp}}^{2}($ Alt $(n))$ exists and satisfies $\gamma_{\mathrm{cp}}^{2}($ Alt $(n))<$ $12 \log _{2}(n)$. The claim of the lemma follows.

Finally we consider the simple sporadic groups together with the Tits group. The next lemma contains some useful tools.

Lemma 2.6. Let $G$ be a group, and $p$ a prime divisor of $|G|$. Let $P$ be a Sylow p-subgroup of $G$.

(a) Suppose that $A \leq G$ contains $P$, and that $\gamma_{\mathrm{cp}}^{A}(G), \gamma_{\mathrm{cp}}^{p}(G)$ and $\gamma_{\mathrm{cp}}^{p}(A)$ all exist. Then $\gamma_{\mathrm{cp}}^{p}(G) \leq \gamma_{\mathrm{cp}}^{A}(G) \gamma_{\mathrm{cp}}^{p}(A)$.

(b) If $G$ is an almost simple group with socle $S,|G: S|$ is not divisible by $p$ and $\gamma_{\mathrm{cp}}^{p}(S)$ exists, then $\gamma_{\mathrm{cp}}^{p}(G) \leq \gamma_{\mathrm{cp}}^{p}(S)$.

(c) Let $N \unlhd G$. If $N \leq N_{G}(P)$ is solvable then $\gamma_{\mathrm{cp}}^{p}(G)=\gamma_{\mathrm{cp}}^{p}(G / N)$.

(d) If $P$ is non-normal (e.g., $G$ is simple) then $N_{G}(P)$ is solvable if and only if for each maximal subgroup $M$ of $G$ such that $|P|$ divides $|M|$, the normalizer of a Sylow p-subgroup of $M$ is solvable.

(e) If $G$ is a product of $n$ arbitrary $p$-subgroups, and $N_{G}(P)$ is solvable then $\gamma_{\mathrm{cp}}^{p}(G) \leq n$.

(f) If $G$ has a normal subgroup $N$ such that $N$ is a product of $n$ arbitrary p-subgroups, $G / N$ is a p-group, and $N_{G}(P)$ is solvable, then $\gamma_{\mathrm{cp}}^{p}(G) \leq n$.

Proof. (a) By assumption, $A$ is a product of $\gamma_{\mathrm{cp}}^{p}(A) A$-conjugates of $N_{A}(P)$, and $G$ is a product of $\gamma_{\mathrm{cp}}^{A}(G) G$-conjugates of $A$. Hence $G$ is a product of $\gamma_{\mathrm{cp}}^{A}(G) \gamma_{\mathrm{cp}}^{p}(A)$ $G$-conjugates of $N_{A}(P)$. The claim now follows from $N_{A}(P) \leq N_{G}(P)$.

(b) Since $S \unlhd G$, we have that $S \cap P$ is a Sylow $p$-subgroup of $S$, and since $|G: S|$ is not divisible by $p$ we get $P=S \cap P$. In [16, Lemma 14], take $m=1$, $X=G$ and $U=N_{G}(P)$. Then $U S=N_{G}(P) S=G$ by Frattini's argument, and 
$U \cap S=N_{S}(P)$. Since $1<N_{S}(P)<S$ we can deduce from [16, Lemma 14], that $G$ is a product of $h=\gamma_{\mathrm{cp}}^{p}(S)$ conjugates of $N_{G}\left(N_{S}(P)\right)$. Now, because $S \unlhd G$, $U=N_{G}(P)$ normalizes $U \cap S=N_{S}(P)$, and hence $N_{G}(P) \leq N_{G}\left(N_{S}(P)\right)$. Using $N_{G}(P) S=G$ and Dedekind's argument we get:

$$
\begin{gathered}
N_{G}\left(N_{S}(P)\right)=N_{G}\left(N_{S}(P)\right) \cap\left(N_{G}(P) S\right)=N_{G}(P)\left(N_{G}\left(N_{S}(P)\right) \cap S\right)= \\
N_{G}(P) N_{S}\left(N_{S}(P)\right)=N_{G}(P) N_{S}(P)=N_{G}(P) .
\end{gathered}
$$

Therefore $G$ is a product of $\gamma_{\mathrm{cp}}^{p}(S)$ conjugates of $N_{G}(P)$. Finally note that the existence of $\gamma_{\mathrm{cp}}^{p}(S)$ implies the solvability of $N_{S}(P)$ which implies, using Schreier's Conjecture, the solvability of $N_{G}(P)$. The claim follows.

(c) For each $A \leq G$ set $\bar{A}:=A N / N$. Then, in general (without assuming $\left.N \leq N_{G}(P)\right)$ we have $\overline{N_{G}(P)}=N_{\bar{G}}(\bar{P})([22,3.2 .8])$. If $N \leq N_{G}(P)$ is solvable then $\overline{N_{G}(P)}=N_{G}(P) / N=N_{\bar{G}}(\bar{P})$, and $N_{G}(P)$ is solvable if and only if $N_{\bar{G}}(\bar{P})$ is solvable. Moreover, in this case $G$ is a product of $k$ conjugates of $N_{G}(P)$ if and only if $\bar{G}$ is a product of $k$ conjugates of $N_{\bar{G}}(\bar{P})$. The claim follows.

(d) This follows from the fact that $N_{M}(P) \leq N_{G}(P)$ for any $M \leq G$, and from the fact that if $P$ is non-normal, then $N_{G}(P)$ is contained in some maximal subgroup of $G$, so $N_{M}(P)=N_{G}(P)$ for some maximal subgroup $M$ of $G$, which contains $P$.

(e) Each $p$-subgroup of $G$ is contained in a Sylow $p$-subgroup of $G$ which is, in turn, contained in its normalizer.

(f) By assumption $N=Q_{1} \cdots Q_{n}$ where each $Q_{i}$ is a $p$-subgroup. Assume, without loss of generality, $Q_{n} \leq P$. We have $G=N P$ because $G / N$ is a $p$-group. Now $G=N P=Q_{1} \cdots Q_{n-1} P$ and the claim follows from (e).

Lemma 2.7. If $S$ is a sporadic simple group or the Tits group then upper bounds on $\gamma_{\mathrm{cp}}^{\mathrm{ss}}(S)$ are given in the Appendix, in Table 1, under the column heading $\gamma_{\mathrm{cp}}^{p}(S) \leq$. It follows, by inspection of the table, that $\gamma_{\mathrm{cp}}^{\mathrm{ss}}(S) \leq 36$.

Proof. The deduction of the upper bounds in Table 1 uses several ingredients. The first one is the detailed information about the maximal subgroups of the sporadic simple groups which is available in [2]. A second ingredient is a basic inequality which relates $\gamma_{\mathrm{cp}}^{p}(G)$ to $\gamma_{\mathrm{cp}}^{p}(A)$ for $A \leq G$. It is stated together with other useful relations in Lemma 2.6 above. A third and crucial ingredient is the possibility to calculate $\gamma_{\mathrm{cp}}^{A}(G)$ for many pairs $(G, A)$ of interest, using the permutation character $1_{A}^{G}$. If the irreducible decomposition of this character in terms of the complex irreducible characters of $G$ is multiplicity free, one can employ a method, developed and implemented in GAP as a tool called "mfer" by T. Breuer, I. Höhler and J. Müller ([7], [6], [15] and [5]), in order to obtain the structure constants of the Hecke algebra of the double cosets of $A$. From these structure constants one can compute $\gamma_{\mathrm{cp}}^{A}(G)$ as explained in [8, Sections 2.1 and 5]. Note that the "mfer" tool can be applied to groups $G$ where $G$ is a simple sporadic group, as well as to some of the groups stored in the TomLib library of [15]. The fourth ingredient are the two results, [8, Theorem 3] (see Lemma 2.4) and the fact that a simple group of Lie type is a product of four Sylow $p$-subgroups, where $p$ is a defining characteristic [17]. Further details on how these four ingredients are used for deducing Table 1 are given in the Appendix. 
2.2. Reduction to special solvable cp-factorizations. In this section we reduce the analysis of $\gamma_{\mathrm{cp}}^{\mathrm{s}}$ to that of $\gamma_{\mathrm{cp}}^{\mathrm{ss}}$ for simple groups.

Lemma 2.8. Let $G=T_{1}^{r_{1}} \times \cdots \times T_{m}^{r_{m}}$ where the $T_{i}$ 's are pairwise non-isomorphic non-abelian simple groups, and $r_{1}, \ldots, r_{m}$ are positive integers. Then

$$
\gamma_{\mathrm{cp}}^{\mathrm{ss}}(G) \leq \max \left\{\gamma_{\mathrm{cp}}^{\mathrm{ss}}\left(T_{1}\right), \ldots, \gamma_{\mathrm{cp}}^{\mathrm{ss}}\left(T_{m}\right)\right\} .
$$

Proof. For each $1 \leq i \leq m$ set $k_{i}:=\gamma_{\mathrm{cp}}^{\mathrm{ss}}\left(T_{i}\right)$. By assumption, for each $1 \leq i \leq m$ there exists $B_{i 1}<T_{i}$ satisfying Definition 2.1 , and $T_{i}=B_{i 1} \cdots B_{i k_{i}}$ where $B_{i j}$ is conjugate in $T_{i}$ to $B_{i 1}$ for all $1 \leq j \leq k_{i}$. Set $k:=\max \left\{k_{1}, \ldots, k_{m}\right\}$. We can assume that the special solvable conjugate factorizations of the $T_{i}$ are all of equal length $k$, since for each $1 \leq i \leq m$ we can add subgroups $B_{i j}$ with $k_{i}+1 \leq j \leq k$, chosen arbitrarily from the conjugates of $B_{i 1}$ in $T_{i}$. Clearly $T_{i}=B_{i 1} \cdots B_{i k}$. We claim that $T_{i}^{r_{i}}=B_{i 1}^{r_{i}} \cdots B_{i k}^{r_{i}}$ is a special solvable conjugate factorization of $T_{i}^{r_{i}}$. It is easy to see that each $B_{i j}^{r_{i}}$ is solvable, being a direct product of solvable groups, and that each $B_{i j}^{r_{i}}$ is conjugate in $T_{i}^{r_{i}}$ to $B_{i 1}^{r_{i}}$ because each $B_{i j}$ is conjugate in $T_{i}$ to $B_{i 1}$. Similarly, $B_{i 1}^{r_{i}}$ is self-normalizing in $T_{i}^{r_{i}}$, because $B_{i 1}$ is self-normalizing in $T_{i}$. In order to verify condition (iii) of Definition 2.3, recall that $A u t\left(T_{i}^{r_{i}}\right) \cong A u t\left(T_{i}\right)^{r_{i}} \rtimes S_{r_{i}}$ where the symmetric group $S_{r_{i}}$ permutes the $r_{i}$ direct factors of $A u t\left(T_{i}\right)^{r_{i}}$ according to its natural action on $\left\{1, \ldots, r_{i}\right\}([27,9.24])$. Thus $S_{r_{i}}$ normalizes $B_{i 1}^{r_{i}}$. Let $\alpha \in$ Aut $\left(T_{i}^{r_{i}}\right)$. We have $\alpha=g\left(\alpha_{1}, \ldots, \alpha_{r_{i}}\right)$ where $\alpha_{j} \in \operatorname{Aut}\left(T_{i}\right), 1 \leq j \leq r_{i}$ and $g \in S_{r_{i}}$. Since $g$ normalizes $B_{i 1}^{r_{i}}$ we get $\left(B_{i 1}^{r_{i}}\right)^{\alpha}=B_{i 1}^{\alpha_{1}} \times \cdots \times B_{i 1}^{\alpha_{r_{i}}}$. Now we can use the fact that $B_{i 1}$ satisfies condition (iii) of Definition 2.3, as a subgroup of $T_{i}$.

Next define for each $1 \leq j \leq k, B_{j}:=\prod_{i=1}^{m} B_{i j}^{r_{i}}$ (a direct product). We have $G=$ $B_{1} \cdots B_{k}$, and again we claim that this is a special solvable conjugate factorization. The proof relies on the previous claim, namely, that $T_{i}^{r_{i}}=B_{i 1}^{r_{i}} \cdots B_{i k}^{r_{i}}$ is a special solvable conjugate factorization, and proceeds in the same way where for showing that $B_{1}$ satisfies condition (iii) of Definition 2.3, we use the fact that $A u t(G)=$ Aut $\left(T_{1}^{r_{1}}\right) \times \cdots \times$ Aut $\left(T_{m}^{r_{m}}\right)$, which follows from the fact that the $T_{i}$ 's are pairwise non-isomorphic non-abelian simple groups ([27, 9.25]). Finally, since $G=B_{1} \cdots B_{k}$ is a special solvable conjugate factorization, we get $\gamma_{\mathrm{cp}}^{\mathrm{ss}}(G) \leq k$ which is what we wanted to prove.

Lemma 2.9. Let $G$ be a group and let $N \unlhd G$. Then $\gamma_{\mathrm{cp}}^{\mathrm{s}}(G) \leq \gamma_{\mathrm{cp}}^{\mathrm{ss}}(N)+\gamma_{\mathrm{cp}}^{\mathrm{s}}(G / N)$.

Proof. We can assume $\gamma_{\mathrm{cp}}^{\mathrm{ss}}(N)<\infty$. Set $t:=\gamma_{\mathrm{cp}}^{\mathrm{s}}(G / N)$. Then, by definition of $\gamma_{\mathrm{cp}}^{\mathrm{s}}(G / N)$, there exists $H \leq G$ and $N \leq H$ such that $H / N$ is solvable, and there exist $t$ subgroups $H_{1}, \ldots, H_{t}$ of $G$, all containing $N$, such that $G / N=\left(H_{1} / N\right) \cdots\left(H_{t} / N\right)$, and $H_{i} / N$ is conjugate to $H / N$ in $G / N$ for each $1 \leq i \leq t$. It follows that $H_{i}$ is conjugate to $H$ in $G$ for all $1 \leq i \leq t$, and $G=H_{1} \cdots H_{t}$.

Set $k:=\gamma_{\mathrm{cp}}^{\mathrm{ss}}(N)$. By definition of $\gamma_{\mathrm{cp}}^{\mathrm{ss}}(N)$, there exists $B \leq N$ satisfying (i)-(iii) in Definition 2.1 and $N=B_{1} \cdots B_{k}$, where each $B_{i}$ is conjugate to $B$ in $N$. We claim that $H=N_{H}(B) N$. First note that both $N_{H}(B)$ and $N$ are subgroups of $H$ so $N_{H}(B) N \leq H$. For the reverse inclusion let $h \in H$ be arbitrary. Since $N \unlhd H$, $h$ acts on $N$ as an automorphism, and therefore, by property (iii) in Definition 2.1, there exists $n \in N$ such that $B^{h}=B^{n}$ from which it follows that $h n^{-1} \in N_{H}(B)$. Hence $h=\left(h n^{-1}\right) n \in N_{H}(B) N$. 
Now $H=N_{H}(B) N$ implies that $H / N=N_{H}(B) N / N \cong N_{H}(B) / B$ (by Definition 2.1.(ii)). But since both $H / N$ and $B$ are solvable, we get that $N_{H}(B)$ is solvable.

For each $1 \leq i \leq t$ let $g_{i} \in G$ be such that $H_{i}=H^{g_{i}}$, and for each $1 \leq j \leq k$ let $\nu_{j} \in N$ be such that $B_{j}=B^{\nu_{j}}$. Using the above we get:

$$
\begin{gathered}
G=H_{1} \cdots H_{t}=H^{g_{1}} \cdots H^{g_{t}}=\left(N_{H}(B) N\right)^{g_{1}} \cdots\left(N_{H}(B) N\right)^{g_{t}}= \\
=\left(N_{H}(B)\right)^{g_{1}} \cdots\left(N_{H}(B)\right)^{g_{t}} N=\left(N_{H}(B)\right)^{g_{1}} \cdots\left(N_{H}(B)\right)^{g_{t}} B_{1} \cdots B_{k}= \\
=\left(N_{H}(B)\right)^{g_{1}} \cdots\left(N_{H}(B)\right)^{g_{t}} B^{\nu_{1}} \cdots B^{\nu_{k}}= \\
=\left(N_{H}(B)\right)^{g_{1}} \cdots\left(N_{H}(B)\right)^{g_{t}}\left(N_{H}(B)\right)^{\nu_{1}} \cdots\left(N_{H}(B)\right)^{\nu_{k}} .
\end{gathered}
$$

Since $N_{H}(B)$ is solvable this implies that $\gamma_{\mathrm{cp}}^{\mathrm{s}}(G) \leq k+t$ as claimed.

Next we apply Lemma 2.9 to the non-abelian socle series (Definition 1.2), whose factors we will denoted $N_{i}:=H_{2 i} / H_{2 i-1}=\operatorname{soc}\left(G / H_{2 i-1}\right)$, and $n_{i}$ will stand for the number of simple non-abelian direct factors of $N_{i}$ for all $1 \leq i \leq t / 2$. Observe that the uniqueness of the non-abelian socle series of $G$ is a consequence of the uniqueness of the solvable radical and the socle of any given group. Moreover, for all $1 \leq i \leq t / 2$ we have $R\left(G / H_{2 i-1}\right)=1$. This is clear for $i=1$, and for $i \geq 2$ we have $G / H_{2 i-1} \cong\left(G / H_{2 i-2}\right) /\left(H_{2 i-1} / H_{2 i-2}\right)$ and now we can use $H_{2 i-1} / H_{2 i-2}=R\left(G / H_{2 i-2}\right)$. Since $R\left(G / H_{2 i-1}\right)=1$ we get that $N_{i}$ is a nontrivial direct product of non-abelian simple groups. As a result, the inclusion $H_{2 i-1} \leq H_{2 i}$ is always strict, while the inclusion $H_{2 i} \leq H_{2 i+1}$ need not be strict. Finally note that the non-solvable length of $G$ is zero if and only if $G$ is solvable.

Proposition 2.10. Let $G$ be a non-trivial group whose non-solvable length is $m:=$ $\lambda(G) \geq 0$. For each $1 \leq i \leq m$ pick a simple non-abelian direct factor $T_{i}$ of $N_{i}$ such that $\gamma_{\mathrm{cp}}^{\mathrm{ss}}\left(T_{i}\right)$ is maximal compared to any other factor of $N_{i}$. Then

$$
\gamma_{\mathrm{cp}}^{\mathrm{s}}(G) \leq 1+\sum_{i=1}^{m} \gamma_{\mathrm{cp}}^{\mathrm{ss}}\left(T_{i}\right)
$$

Proof. By induction on $m \geq 0$. If $m=0$ then $G$ is solvable and so $\gamma_{\mathrm{cp}}^{\mathrm{s}}(G)=1$. Suppose $m>0$. Then $\gamma_{\mathrm{cp}}^{\mathrm{s}}(G) \leq \gamma_{\mathrm{cp}}^{\mathrm{s}}(G / R(G))$. In fact equality holds since if $G=A_{1} \ldots A_{k}$ is a solvable cp-factorization then so is $G=\left(A_{1} R(G)\right) \ldots\left(A_{k} R(G)\right)$. Moreover, if $R(G)=H_{1} \leq \ldots \leq H_{t}=G$ is the non-abelian socle series of $G$, then $1=H_{1} / R(G) \leq \ldots \leq H_{t} / R(G)=G / R(G)$ is the non-abelian socle series of $G / R(G)$. We have $R(G / R(G))=1$ and $N_{1}=H_{2} / R(G)$. Hence, by Lemma 2.9 we have $\gamma_{\mathrm{cp}}^{\mathrm{s}}(G)=\gamma_{\mathrm{cp}}^{\mathrm{s}}(G / R(G)) \leq \gamma_{\mathrm{cp}}^{\mathrm{ss}}\left(N_{1}\right)+\gamma_{\mathrm{cp}}^{\mathrm{s}}\left((G / R(G)) /\left(H_{2} / R(G)\right)\right)$. Using $(G / R(G)) /\left(H_{2} / R(G)\right) \cong G / H_{2}$, we obtain $\gamma_{\mathrm{cp}}^{\mathrm{s}}(G) \leq \gamma_{\mathrm{cp}}^{\mathrm{ss}}\left(N_{1}\right)+\gamma_{\mathrm{cp}}^{\mathrm{s}}\left(G / H_{2}\right)$. By Lemma $2.8, \gamma_{\mathrm{cp}}^{\mathrm{ss}}\left(N_{1}\right) \leq \gamma_{\mathrm{cp}}^{\mathrm{ss}}\left(T_{1}\right)$, and since the non-abelian socle length of $G / H_{2}$ is $m-1$, we have by induction $\gamma_{\mathrm{cp}}^{\mathrm{s}}\left(G / H_{2}\right) \leq 1+\sum_{i=2}^{m} \gamma_{\mathrm{cp}}^{\mathrm{ss}}\left(T_{i}\right)$. The claim follows.

2.3. Proof of Theorem 1.3. By Proposition 2.10 we have

$$
\gamma_{\mathrm{cp}}^{\mathrm{s}}(G) \leq 1+\sum_{i=1}^{\lambda(G)} \gamma_{\mathrm{cp}}^{\mathrm{ss}}\left(T_{i}\right) \leq 1+\lambda(G) \max \left\{\gamma_{\mathrm{cp}}^{\mathrm{ss}}\left(T_{i}\right)\right\}_{1 \leq i \leq \lambda(G)}
$$

Combining the results of Lemma 2.4, Lemma 2.5 and Lemma 2.7 we get:

$$
\max \left\{\gamma_{\mathrm{cp}}^{\mathrm{ss}}\left(T_{i}\right)\right\}_{1 \leq i \leq \lambda(G)}=\max \left\{36,12 \log _{2} n\right\}
$$


where $n$ is the largest integer for which there exists $1 \leq i \leq \lambda(G)$ such that $T_{i} \cong \operatorname{Alt}(n)$, and $n=2$ if no such $i$ exists.

\section{AN UPPER BOUND ON THE NON-SOLVABLE LENGTH}

In this section we derive an upper bound on the non-solvable length of a nonsolvable group (we keep the notation introduced in Subsection 2.2 immediately before Proposition 2.10). This result can then be applied to Theorem 1.3. Recall that for any group $H$ there exists the least integer $n$, customarily denoted $\mu(H)$, such that $H$ embeds in the symmetric group $S_{n}$. In other words, $\mu(H)$ is the minimal degree of a faithful permutation representation of $H$. We will make use of the following properties of this quantity. If $H_{1} \leq H$ then $\mu\left(H_{1}\right) \leq \mu(H)$ (immediate from the definition). If $N=T_{1} \times \cdots \times T_{k}$ where $T_{i}$ simple non-abelian for each $1 \leq i \leq k$ then $\mu(N)=\sum_{i=1}^{k} \mu\left(T_{i}\right)$ ([13, Theorem 3.1]). If $N \unlhd H$ and $R(H / N)=1$ then $\mu(H / N) \leq \mu(H)$ ([21, Theorem 1]). If $T$ is simple non-abelian then $\mu(T) \geq 5$ (since all subgroups of $S_{n}$ are solvable if $n<5$ ).

Lemma 3.1. Let $G$ be a non-solvable group and let $R(G)=H_{1} \leq \ldots \leq H_{t}=G$ be the non-abelian socle series of $G$. Then, for each $1<i \leq t / 2, \mu\left(G / H_{2 i-1}\right) \leq n_{i-1}$.

Proof. It is clearly sufficient to prove the statement for the case $i=2$. So we will prove $\mu\left(G / H_{3}\right) \leq n_{1}$. Since $G / H_{1}$ has a trivial solvable radical it acts faithfully by conjugation on $N_{1}=\operatorname{soc}\left(G / H_{1}\right)=H_{2} / H_{1}$ and so embeds in $\operatorname{Aut}\left(N_{1}\right)$. Now $N_{1}=T_{1}^{r_{1}} \times \cdots \times T_{m}^{r_{m}}$ where the $T_{i}$ 's are pairwise non-isomorphic non-abelian simple groups, and $r_{1}, \ldots, r_{m}$ are positive integers $\left(\sum_{i=1}^{m} r_{i}=n_{1}\right)$. We have $\operatorname{Aut}\left(N_{1}\right)=$ Aut $\left(T_{1}^{r_{1}}\right) \times \cdots \times \operatorname{Aut}\left(T_{m}^{r_{m}}\right)$ and Aut $\left(T_{i}^{r_{i}}\right) \cong \operatorname{Aut}\left(T_{i}\right)^{r_{i}} \rtimes S_{r_{i}}$ (see proof of Lemma 2.8). Now, the image of $G / H_{1}$ in $\operatorname{Aut}\left(N_{1}\right)$ contains $\operatorname{Inn}\left(T_{1}^{r_{1}}\right) \times \cdots \times \operatorname{Inn}\left(T_{m}^{r_{m}}\right)=$ $\left(\operatorname{Inn}\left(T_{1}\right)\right)^{r_{1}} \times \cdots \times\left(\operatorname{Inn}\left(T_{m}\right)\right)^{r_{m}}$ which is, in fact, the image of $N_{1}$ so

$$
\begin{aligned}
\left(G / H_{1}\right) / N_{1}=\left(G / H_{1}\right) / & \left(H_{2} / H_{1}\right) \cong G / H_{2} \precsim \operatorname{Aut}\left(N_{1}\right) / \prod_{i=1}^{m}\left(\operatorname{Inn}\left(T_{i}\right)\right)^{r_{i}} \\
& \cong \prod_{i=1}^{m} \operatorname{Out}\left(T_{i}\right)^{r_{i}} \rtimes S_{r_{i}},
\end{aligned}
$$

where $\prod_{i=1}^{m}$ is direct, Out $\left(T_{i}\right):=\operatorname{Aut}\left(T_{i}\right) / \operatorname{Inn}\left(T_{i}\right)$, and $\precsim$ denotes embedding. Set $A:=\prod_{i=1}^{m} \operatorname{Out}\left(T_{i}\right)^{r_{i}} \rtimes S_{r_{i}}, B:=\prod_{i=1}^{m} \operatorname{Out}\left(T_{i}\right)^{r_{i}}$ and $\widetilde{S}:=\prod_{i=1}^{m} S_{r_{i}} \leq S_{n_{1}}$. We have $A=B S$, and $B \unlhd A$. Furthermore, $B$ is solvable by Schreier's conjecture. Hence $B \leq R(A)$. Therefore $A / R(A)=S R(A) / R(A) \cong S /(S \cap R(A))$. Since $R(A / R(A))=1$ we have $R(S /(S \cap R(A)))=1$. Thus, by [21, Theorem 1],

$$
\mu(A / R(A))=\mu(S /(S \cap R(A))) \leq \mu(S) \leq \mu\left(S_{n_{1}}\right)=n_{1} .
$$

Now, identifying $G / H_{2}$ with its embedding in $A$, we have that $\left(G / H_{2}\right) R(A) / R(A)$ is a subgroup of $A / R(A)$ and so $\mu\left(\left(G / H_{2}\right) R(A) / R(A)\right) \leq \mu(A / R(A))$. On the other hand,

$$
\left(G / H_{2}\right) R(A) / R(A) \cong\left(G / H_{2}\right) /\left(\left(G / H_{2}\right) \cap R(A)\right) .
$$

Set $D:=\left(G / H_{2}\right) \cap R(A) \leq R\left(G / H_{2}\right)$. By an isomorphism theorem we have $\left(\left(G / H_{2}\right) / D\right) /\left(R\left(G / H_{2}\right) / D\right) \cong\left(G / H_{2}\right) / R\left(G / H_{2}\right)$. Hence, by [21, Theorem 1], 
we have

$$
\begin{aligned}
\mu\left(\left(G / H_{2}\right) / R\left(G / H_{2}\right)\right) & \leq \mu\left(\left(G / H_{2}\right) / D\right)=\mu\left(\left(G / H_{2}\right) R(A) / R(A)\right) \\
& \leq \mu(A / R(A)) \leq n_{1} .
\end{aligned}
$$

On the other hand

$$
\left(G / H_{2}\right) / R\left(G / H_{2}\right)=\left(G / H_{2}\right) /\left(H_{3} / H_{2}\right) \cong G / H_{3},
$$

and the claim $\mu\left(G / H_{3}\right) \leq n_{1}$ follows.

Lemma 3.2. Let $G$ be a non-solvable group, and let $m$ be the non-solvable length of $G$. Then $5 n_{i} \leq n_{i-1}$ for all $2 \leq i \leq m$.

Proof. Let $R(G)=H_{1} \leq \ldots \leq H_{t}=G$ be the non-abelian socle series of $G$. By the preceding remarks, $N_{i}=\operatorname{soc}\left(G / H_{2 i-1}\right)=T_{i 1} \times \cdots \times T_{i n_{i}}$ for all $1 \leq i \leq m$, where each $T_{i j}$ is a non-abelian simple group. We have:

$$
\mu\left(N_{i}\right)=\sum_{j=1}^{n_{i}} \mu\left(T_{i j}\right) \geq 5 n_{i} .
$$

On the other hand, by Lemma $3.1, \mu\left(N_{i}\right) \leq \mu\left(G / H_{2 i-1}\right) \leq n_{i-1}$. Thus $5 n_{i} \leq n_{i-1}$ for all $2 \leq i \leq m$.

Proof of Theorem 1.4. By the previous lemma and using the notation above, $5 n_{i} \leq n_{i-1}$ for all $2 \leq i \leq m$. Since $n_{m} \geq 1$ we get by induction, $n_{i} \geq 5^{m-i}$ for all $1 \leq i \leq m$, and hence the total number $k$ of non-abelian composition factors of $G$ satisfies

$$
k=\sum_{i=1}^{m} n_{i} \geq \sum_{i=1}^{m} 5^{m-i}=\sum_{i=0}^{m-1} 5^{i}=\frac{5^{m}-1}{4} .
$$

This is equivalent to $5^{m} \leq 4 k+1$ and the claim of the theorem follows.

\section{Nilpotent cp-FaCtorizations}

4.1. Nilpotent cp-factorizations of solvable groups. In this subsection we prove Theorem 1.6. Using the special properties of Carter subgroups we are able to reduce to the case where $G$ is an affine primitive group. For this case we rely on the following theorem.

Theorem $4.1([18])$. Let $G$ be an affine primitive permutation group with a nontrivial point stabilizer $H$. Then $G$ is a product of at most $1+c_{\mathrm{ca}} \log _{2}|G: H|$ conjugates of $H$, where $0<c_{\text {ca }} \leq 3 / \log _{2} 5<1.3$ is a universal constant.

Recall that $C \leq G$ is a Carter subgroup of $G$ if $C$ is nilpotent and self normalizing. The following lemmas collect various properties of Carter subgroups which are required for the proof of Theorem 1.6.

Lemma 4.2. Let $G$ be a solvable group. Then

(a) There exists a Carter subgroup of $G$.

(b) There is a unique conjugacy class of Carter subgroups in $G$.

(c) If $C$ is a Carter subgroup of $G$ then $C$ is a maximal nilpotent subgroup of $G$, that is, if $C<H \leq G$, then $H$ is not nilpotent.

(d) If $C$ is a Carter subgroup of $G$ and $N \unlhd G$ then $C N / N$ is a Carter subgroup of $G / N$. 
(e) If $C$ is a Carter subgroup of $G$ then $C$ is Carter subgroup of $H$ for any $C \leq H \leq G$.

Proof. For (a)-(d) see [9] and [20, Theorem 12.2(b) and Lemma 12.3]. For (e) note that since $C$ is self-normalizing in $G$ it is self-normalizing in any subgroup of $G$ containing $C$.

Lemma 4.3. Let $G$ be a solvable group and let $C$ be a Carter subgroup of $G$. Then $G$ is a product of conjugates of $C$.

Proof. It is enough to show that $C^{G}=G$. Set $N:=C^{G}$. Note that $C$ is a Carter subgroup of $N$. For any $g \in G$ we have $C^{g} \leq N$ is a self normalizing nilpotent subgroup of $N$. Hence $C^{g}$ is a Carter subgroup of $N$ and hence there exists $n \in N$ such that $C^{g}=C^{n}$. It follows that $g n^{-1}$ normalizes $C$ and therefore $g n^{-1} \in C \leq N$. Hence $g \in N$ implying $G=N$.

Definition 4.4. Let $G$ be a solvable group. We denote by $\gamma_{\mathrm{cp}}^{\mathrm{c}}(G)$ the minimal length of a cp-factorization of $G$ by a Carter subgroup.

Note that a nilpotent group $G$ is equal to its own Carter subgroup and hence, for $G$ nilpotent, $\gamma_{\mathrm{cp}}^{\mathrm{c}}(G)=1$. Clearly $\gamma_{\mathrm{cp}}^{\mathrm{n}}(G) \leq \gamma_{\mathrm{cp}}^{\mathrm{c}}(G)$.

Lemma 4.5. Let $G$ be a solvable group and let $C$ be a Carter subgroup of $G$. Let $N \unlhd G$ be such that $N$ is contained in $C$. Then $\gamma_{\mathrm{cp}}^{\mathrm{c}}(G)=\gamma_{\mathrm{cp}}^{\mathrm{c}}(G / N)$.

Proof. It is clear that if $N$ is contained in $C$ then it is contained in every conjugate of $C$. Suppose that $G=C_{1} \cdots C_{k}$ where $C_{i}$ is a conjugate of $C$ for all $1 \leq i \leq k$. Then $\bar{G}=\overline{C_{1}} \cdots \overline{C_{k}}$, where, for any $A \leq G$ we denote $\bar{A}:=A N / N$, and each $\overline{C_{i}}$ is a Carter subgroup of $\bar{G}$. Conversely, if $\bar{G}=\overline{C_{1}} \cdots \overline{C_{k}}$, where the $\overline{C_{i}}$ are Carter subgroups of $\bar{G}$, then, by assumption, the full preimage of $\overline{C_{i}}$ in $G$ is a Carter subgroup $C_{i}$ of $G$ and we can conclude that $G=C_{1} \cdots C_{k}$. The claim follows.

Proof of Theorem 1.6. The proof is by induction on $|G|$. Let $C$ be a Carter subgroup of $G$. If $G$ is nilpotent then $G=C, \gamma_{\mathrm{cp}}^{\mathrm{c}}(G)=1$, and the claim clearly holds. Hence we can assume that $G$ is non-nilpotent. Let $N$ be a minimal normal subgroup of $G$. For any $A \leq G$ denote $\bar{A}:=A N / N$. Then $\bar{G}=\overline{C_{1}} \cdots \overline{C_{k}}$, where each $\overline{C_{i}}$ is a Carter subgroup of $\bar{G}$, and $k=\gamma_{\mathrm{cp}}^{\mathrm{c}}(\bar{G})$. By Lemma $4.2(\mathrm{~d})$, the full preimage of $\overline{C_{i}}$ in $G$ is $C_{i} N$ where $C_{i}$ is a Carter subgroup of $G$, and we get $G=C_{1} \cdots C_{k} N$. If $k>1, C_{k} N$ is proper in $G$. By Lemma $4.2(\mathrm{e}) C_{k}$ is a Carter subgroup of $C_{k} N$ and hence we get by induction that $C_{k} N$ is a product of $\gamma_{\mathrm{cp}}^{\mathrm{c}}\left(C_{k} N\right) \leq c_{\mathrm{ca}} \log _{2}\left(\left|C_{k} N: C_{k}\right|\right)+1$ conjugates of $C_{k}$. Since $C_{k}$ is conjugate to $C$ and $N$ is normal we have $\left|C_{k} N: C_{k}\right|=|C N: C|$. Therefore

$$
\begin{aligned}
& \gamma_{\mathrm{cp}}^{\mathrm{c}}(G) \leq k-1+c_{\mathrm{ca}} \log _{2}\left(\left|C_{k} N: C_{k}\right|\right)+1=\gamma_{\mathrm{cp}}^{\mathrm{c}}(\bar{G})+c_{\mathrm{ca}} \log _{2}(|C N: C|) \\
& \leq 1+c_{\mathrm{ca}} \log _{2}(|G / N: C N / N|)+c_{\mathrm{ca}} \log _{2}(|C N: C|)=1+c_{\mathrm{ca}} \log _{2}(|G: C|),
\end{aligned}
$$

and the claim is proved. Hence we can assume $k=1$.

In this case we have $G=C N$, where $C$ is a Carter subgroup of $G$ and $N$ is a minimal normal subgroup of $G$. Since $G$ is solvable, $N$ is elementary abelian and in particular, $|N|=p^{n}$ for some prime $p$ and some positive integer $n$. Suppose that $C$ contains a non-trivial normal subgroup $L$ of $G$. By Lemma $4.5, \gamma_{\mathrm{cp}}^{\mathrm{c}}(G)=\gamma_{\mathrm{cp}}^{\mathrm{c}}(G / L)$ and $G / L=(C / L) N$ and $N$ is minimal normal in $G / L$. Thus we can assume that $C$ is core-free. Under this assumption $C_{G}(N)=N$. Indeed, $N \leq C_{G}(N)$ because 
$N$ is abelian so by Dedekind's law, $C_{G}(N)=C_{G}(N) \cap(N C)=N\left(C_{G}(N) \cap C\right)$. Since $C_{G}(N) \unlhd G$ we get that $C$ normalizes $C_{G}(N) \cap C$. Moreover, $N$ centralizes $C_{G}(N)$ hence it normalizes $C_{G}(N) \cap C$. Thus we proved that $C_{G}(N) \cap C \unlhd G$. Since $C$ is core-free, we get $C_{G}(N) \cap C=1$ and $C_{G}(N)=N$. Finally, since $G$ is solvable, $G$ is primitive iff it has a self-centralizing minimal normal subgroup ([12, Proposition A.15.8(b)]). Thus we can conclude that $G$ is primitive and $C$ is maximal and non-normal. Now apply Theorem 4.1, with $H=C$.

4.2. Nilpotent cp-factorizations of non-solvable groups. This subsection is devoted to the proof of Theorem 1.7. This requires a readjustment of some of the results in Subsections 2.1 and 2.2 .

Lemma 4.6. Let $S$ be a simple non-abelian group. Then there exist a subgroup $A<$ $S$ and two universal real positive constants $c$ and $u$ such that $S$ has a special solvable cp-factorization by at most $c \log _{2} \log _{2}|S|$ conjugates of $A$, and $|S| \leq|S: A|^{u}$.

Proof. It is sufficient to prove the claim separately for $S$ a simple group of Lie type, $S$ a simple alternating group, and $S$ a sporadic simple group or the Tits group.

1. $S$ is a simple group of Lie type. Take $A$ to be a Borel subgroup of $S$. Since (see Lemma 2.4) $S$ is a product of three conjugates of $A$, and since $|S| \geq 60$, choosing $c$ to satisfy $c \geq 3 / \log _{2} \log _{2} 60$ gives that $S$ is a product of at most $c \log _{2} \log _{2}|S|$ conjugates of $A$. Moreover, let $p$ be a defining characteristic of $S$ and let $U$ be a Sylow $p$-subgroup of $S$. Then, by [17], $S$ is a product of 4 conjugates of $U$, and so $|S| \leq|U|^{4}$. On the other hand $|S: A| \geq|U|$. To see this, use the fact that $S$ is a group with a split $B N$-pair and that by [10, Proposition 2.5.13] $A \dot{w}_{0} A=U \dot{w}_{0} A$ with uniqueness of expression on the r.h.s (see [10] Chapter 2 for notation). Then we have $|S| \geq\left|A \dot{w}_{0} A\right|=|U||A|$, implying $|S: A| \geq|U|$. Combining the two claims gives $|S| \leq|S: A|^{4}$.

2. $S \cong \operatorname{Alt}(n)$ where $n \geq 5$. Since $|S|=n ! / 2$ there exists $c>0$ such that for all $n \geq 5$ we have $c \log _{2} \log _{2}|S| \leq 12 \log _{2}(n)$. Taking $A$ to be a Sylow 2 -subgroup of $S$ we get, by Lemma 2.5, that $S$ has a special solvable cp-factorization by at most $c \log _{2} \log _{2}|S|$ conjugates of $A$. Furthermore we prove that $|S| \leq|S: A|^{2}$. This is equivalent to $2|A|^{2} \leq n$ !, where $|A|$ is equal to the maximal power of 2 dividing $n ! / 2$. First one verifies directly that $2|A|^{2} \leq n$ ! for $n=5,6,7$. Then, by [11, Exercise 2.6.8], $|A|<2^{n-1}$. Since $2^{2 n-1} \leq n$ ! holds, by easy induction, for all $n \geq 8$, the proof is finished.

3. $S$ is a sporadic simple group or the Tits group. This is a collection of 27 groups and the claim follows from the fact that it is a finite collection.

Lemma 4.7. Let $G=T_{1}^{r_{1}} \times \cdots \times T_{m}^{r_{m}}$ where the $T_{i}$ 's are pairwise non-isomorphic non-abelian simple groups, and $r_{1}, \ldots, r_{m}$ are positive integers. Then there exists a solvable subgroup $A$ of $G$ such that

$$
\gamma_{\mathrm{cp}}^{A}(G) \leq \max \left\{\gamma_{\mathrm{cp}}^{\mathrm{ss}}\left(T_{1}\right), \ldots, \gamma_{\mathrm{cp}}^{\mathrm{ss}}\left(T_{m}\right)\right\},
$$

and $|G| \leq|G: A|^{u}$, where the constant $u$ is the same as in Lemma 4.6. 
Proof. Taking $A=B_{1}$ where $B_{1}$ is defined in the proof of Lemma 2.8, we get $\gamma_{\mathrm{cp}}^{A}(G) \leq \max \left\{\gamma_{\mathrm{cp}}^{\mathrm{ss}}\left(T_{1}\right), \ldots, \gamma_{\mathrm{cp}}^{\mathrm{ss}}\left(T_{m}\right)\right\}$. Moreover

$$
\begin{gathered}
|G|=\prod_{i=1}^{m}\left|T_{i}\right|^{r_{i}} \leq \prod_{i=1}^{m}\left(\left|T_{i}: B_{i 1}\right|^{u}\right)^{r_{i}}=\left(\prod_{i=1}^{m}\left(\left|T_{i}: B_{i 1}\right|^{r_{i}}\right)\right)^{u} \\
=\left(\prod_{i=1}^{m}\left|T_{i}^{r_{i}}: B_{i 1}^{r_{i}}\right|\right)^{u}=\left|\prod_{i=1}^{m} T_{i}^{r_{i}}: \prod_{i=1}^{m} B_{i 1}^{r_{i}}\right|^{u}=\left|G: B_{1}\right|^{u}=|G: A|^{u} .
\end{gathered}
$$

Notation 1. Let $G$ be a group. If $G$ is non-solvable then $|G|_{\text {nab }}$ will denote the product of the orders of all non-abelian composition factors of $G$ appearing in a composition series of $G$. If $G$ is solvable then $|G|_{n a b}:=1$.

Lemma 4.8. Let $G$ be a group and let $N \unlhd G$ be such that $\gamma_{\mathrm{cp}}^{\mathrm{ss}}(N)<\infty$. Let $B<N$ be such that $N$ has a special solvable cp-factorization by $\gamma_{\mathrm{cp}}^{\mathrm{ss}}(N)$ conjugates of $B$, and let $H \leq G$ be such that $G / N$ has a solvable cp-factorization by $\gamma_{\mathrm{cp}}^{\mathrm{s}}(G / N)$ conjugates of $H / N$. Assume further that $|N|_{n a b} \leq|N: B|^{u}$ and $|G / N|_{n a b} \leq|G / N: H / N|^{u}$ for some real positive $u$. Set $A:=N_{H}(B)$. Then $A$ is solvable, $\gamma_{\mathrm{cp}}^{A}(G) \leq \gamma_{\mathrm{cp}}^{\mathrm{ss}}(N)+$ $\gamma_{\mathrm{cp}}^{\mathrm{s}}(G / N)$, and $|G|_{n a b} \leq|G: A|^{u}$.

Proof. In view of Lemma 2.9 and its proof, $A$ is solvable and $\gamma_{\mathrm{cp}}^{A}(G) \leq \gamma_{\mathrm{cp}}^{\mathrm{ss}}(N)+$ $\gamma_{\mathrm{cp}}^{\mathrm{s}}(G / N)$. It remains to prove $|G|_{n a b} \leq|G: A|^{u}$. By the proof of Lemma 2.9, $H=A N$. Moreover, using (ii) of Definition 2.1, we have $A \cap N=N_{N}(B)=B$. Using this and the isomorphism theorems gives

$$
\begin{gathered}
|G: A|=|G: H||H: A|=|G: H||A N: A| \\
=|G: H||N: B|=|G / N: H / N||N: B| .
\end{gathered}
$$

By our assumptions, $|G|_{n a b}=|G / N|_{n a b}|N|_{n a b} \leq|G / N: H / N|^{u}|N: B|^{u}$ and therefore, by the above $|G|_{n a b} \leq|G: A|^{u}$.

The next result is a restatement of Proposition 2.10 with an additional conclusion. We use the notation of Subsection 2.2.

Proposition 4.9. Let $G$ be a non-trivial group whose non-solvable length is $m:=$ $\lambda(G) \geq 0$. For each $1 \leq i \leq m$ pick a simple non-abelian direct factor $T_{i}$ of $N_{i}$ such that $\gamma_{\mathrm{cp}}^{\mathrm{ss}}\left(T_{i}\right)$ is maximal compared to any other factor of $N_{i}$. Then there exists a solvable subgroup $A$ of $G$ such that $\gamma_{\mathrm{cp}}^{A}(G) \leq 1+\sum_{i=1}^{m} \gamma_{\mathrm{cp}}^{\mathrm{ss}}\left(T_{i}\right)$ and $|G|_{n a b} \leq|G: A|^{u}$, where the constant $u$ is the same as the one in Lemma 4.6.

Proof. If $m=0$ then $G$ is solvable, $A=G,|G|_{n a b}=1$ and the claim holds. Suppose that $m>0$. Let $N:=N_{1} \unlhd G / R(G)$. In order to simplify notation, assume without loss of generality that $R(G)=1$, so $N \unlhd G$. Note that $|N|=|N|_{n a b}$. Hence, by Lemma 4.7, there exists $B<N\left(B_{1}\right.$ in Lemma 4.7) such that $N$ has a special solvable cp-factorization by $\gamma_{\mathrm{cp}}^{\mathrm{ss}}(N)$ conjugates of $B$, and $|N|_{n a b} \leq|N: B|^{u}$. Since the non-solvable length of $G / N$ is $m-1$ we get, by induction assumption, that $G / N$ is a product of at most $1+\sum_{i=2}^{m} \gamma_{\mathrm{cp}}^{\mathrm{ss}}\left(T_{i}\right)$ conjugates of a solvable $H / N \leq G / N$ and $|G / N|_{n a b} \leq|G / N: H / N|^{u}$. Now the claim follows from Lemma 4.8. 
Proof of Theorem 1.7. By Theorem 1.6 we can assume that $G$ is non-solvable, and therefore $m:=\lambda(G) \geq 1$. By Proposition 4.9 there exists a solvable subgroup $A_{1}$ of $G$ such that $\gamma_{\mathrm{cp}}^{A_{1}}(G) \leq 1+\sum_{i=1}^{m} \gamma_{\mathrm{cp}}^{\mathrm{ss}}\left(T_{i}\right)$, and $|G|_{n a b} \leq\left|G: A_{1}\right|^{u}$, for some real universal positive $u$. By Lemma 4.6, there exists a universal real positive constant $c$ such that $\gamma_{\mathrm{cp}}^{\mathrm{ss}}\left(T_{i}\right) \leq c \log _{2} \log _{2}\left|T_{i}\right|$ for all $1 \leq i \leq m$. Hence

$$
\begin{aligned}
\gamma_{\mathrm{cp}}^{A_{1}}(G) & \leq 1+\sum_{i=1}^{m} c \log _{2} \log _{2}\left|T_{i}\right| \leq 1+c \sum_{i=1}^{m} \log _{2} \log _{2}|G|_{\text {nab }} \\
& =c m \log _{2} \log _{2}|G|_{n a b} .
\end{aligned}
$$

By Theorem 1.4, $m \leq \log _{5}(4 k+1)$ where $k$ is the number of non-abelian composition factors of $G$. Since each non-abelian composition factor is of order at least 60 , we get that $|G|_{n a b} \geq 60^{k}>2^{5 k}$, which implies $k<\frac{1}{5} \log _{2}|G|_{n a b}$. Thus

$$
m \leq \log _{5}(4 k+1)<\log _{5}\left(\frac{4}{5} \log _{2}|G|_{n a b}+1\right)<\log _{2} \log _{2}|G|_{n a b} .
$$

Inserting in the previous inequality we obtain

$$
\gamma_{\mathrm{cp}}^{A_{1}}(G) \leq c\left(\log _{2} \log _{2}|G|_{n a b}\right)^{2} \leq c\left(\log _{2} u+\log _{2} \log _{2}\left|G: A_{1}\right|\right)^{2} .
$$

Now, by Theorem 1.6, $\gamma_{\mathrm{cp}}^{A}\left(A_{1}\right) \leq 1+c_{\mathrm{ca}} \log _{2}\left|A_{1}: A\right|$ where $A$ is a Carter subgroup of $A_{1}$ and $c_{\mathrm{ca}}$ is a universal real positive constant. Since $\gamma_{\mathrm{cp}}^{A}(G) \leq \gamma_{\mathrm{cp}}^{A}\left(A_{1}\right) \gamma_{\mathrm{cp}}^{A_{1}}(G)$, $\left|A_{1}: A\right|<|G: A|$ and $\left|G: A_{1}\right| \leq|G: A|$, the claim of the theorem follows easily for a suitable choice of the constant $c_{u s}$.

Remark 4.10. Using results from Subsections 2.1 and 4.2, and from Table 1, it is possible to give an explicit upper bound on $c_{u s}$.

\section{APPENDIX}

Table 1 lists, for each sporadic simple group $S$ including the Tits group ${ }^{2} F_{4}(2)^{\prime}$, an upper bound on $\gamma_{\mathrm{cp}}^{p}(S)$ (column heading $\gamma_{\mathrm{cp}}^{p}(S) \leq$ ) for a specified prime $p$. Under column heading $p^{\alpha}$ the maximal power of $p$ dividing $|S|$ is given. Under the column heading $A$ we specify a subgroup $A<S$ on which the bound is based, using ATLAS notation ([2]). We have verified, using Lemma $2.6(\mathrm{~d})$ and sometimes a MAGMA computation ([4]), that $N_{S}(P)$, the normalizer in $S$ of some Sylow $p$-subgroup $P$ of $S$ is solvable. For each $A$ in the table we have $\gamma_{\mathrm{cp}}^{A}(S)=3$ - this was verified using the "mfer" tool [6] (see Subsection 2.1). In all cases, with a few exceptions detailed below (all associated with $p=2$ ), $A$ contains a Sylow $p$-subgroup of $S$. For $S=M_{11}, J_{1}$, we have $P \leq A \leq N_{S}(P)$ so the bound is exact and $\gamma_{\mathrm{cp}}^{p}(S)=3$. For the other cases the bound is derived using Lemma 2.6 (a) and a bound on $\gamma_{\mathrm{cp}}^{p}(A)$ (when $\gamma_{\mathrm{cp}}^{p}(S) \leq 9$, we have $\gamma_{\mathrm{cp}}^{p}(A)=3$ ). The determination of the bound on $\gamma_{\mathrm{cp}}^{p}(A)$ uses a variety of means: Lemma 2.4, information on subgroups of $A$ from [2], an application of the "mfer" tool to $A$, and previous results from the table. For the $S=B$, where the bound is 12 , we have deduced $\gamma_{\mathrm{cp}}^{2}(A) \leq 4$ from [17]. In this as well as in the case of $S=M$, the argument relies on Lemma 2.6 (e), (f), and hence $A$ need not contain a Sylow 2-subgroup of $S$.

Remarks for Table 1:

(1) A Sylow 2-subgroup of $A$ is self-normalizing of index 3 , hence $\gamma_{\mathrm{cp}}^{2}(A)=3$. 
(2) A Tomlib mfer calculation shows that $L_{2}(16)$ is a product of three Sylow 5 -subgroup normalizers (structure $D_{30}$ ). Hence $L_{2}(16): 2$ is a product of three Sylow 5-subgroup normalizers (structure $D_{10} \times S_{3}$ ).

${ }^{(3)},{ }^{(4)},{ }^{(6)} A$ is a group of Lie type hence it is a product of three Sylow 2-subgroup normalizers. A MAGMA computation shows that the Sylow 2-subgroup of $A$ is selfnormalizing. Therefore $S$ is a product of nine conjugates of a 2-subgroup and hence it is a product of nine Sylow 2-subgroup normalizers.

(5) $2 . H S .2$ is a central extension of $H S .2$ hence the order 2 center is contained in the Sylow 11-subgroup normalizer of 2.HS.2.

\begin{tabular}{|c|c|c|c|c|}
\hline$S$ & $A$ & $p^{\alpha}$ & $\gamma_{\mathrm{cp}}^{p}(S) \leq$ & Remarks \\
\hline$M_{11}$ & $11: 5$ & 11 & 3 & \\
\hline$M_{12}$ & $M_{11}$ & 11 & 9 & \\
\hline$J_{1}$ & $2^{3}: 7: 3$ & $2^{3}$ & 3 & \\
\hline$M_{22}$ & $L_{2}(11)$ & 11 & 9 & \\
\hline$J_{2}$ & $U_{3}(3)$ & $3^{3}$ & 9 & \\
\hline$M_{23}$ & $M_{11}$ & 11 & 9 & \\
\hline${ }^{2} F_{4}(2)^{\prime}$ & $2^{2} \cdot\left[2^{8}\right]: S_{3}$ & $2^{11}$ & 9 & $(1)$ \\
\hline$H S$ & $M_{11}$ & 11 & 9 & \\
\hline$J_{3}$ & $L_{2}(16): 2$ & 5 & 9 & $(2)$ \\
\hline$M_{24}$ & $2^{6}:\left(L_{3}(2) \times S_{3}\right)$ & $2^{10}$ & 9 & \\
\hline$M^{c} L$ & $U_{4}(3)$ & $3^{6}$ & 9 & \\
\hline$H e$ & $S_{4}(4): 2$ & $2^{10}$ & 9 & $(3)$ \\
\hline$R u$ & $2 F_{4}(2)$ & $2^{14}$ & 9 & $(4)$ \\
\hline$S u z$ & $2_{-}^{1+6} \cdot U_{4}(2)$ & $2^{13}$ & 9 & \\
\hline$O^{\prime} N$ & $L_{3}(7): 2$ & $7^{3}$ & 9 & \\
\hline$C o_{3}$ & $2 . S_{6}(2)$ & $2^{10}$ & 9 & \\
\hline$C o_{2}$ & $2_{+}^{1+8}: S_{6}(2)$ & $2^{18}$ & 9 & \\
\hline$F i_{22}$ & $O_{7}(3)$ & $3^{9}$ & 9 & \\
\hline$H N$ & $2 . H S \cdot 2$ & 11 & 27 & $(5)$ \\
\hline$L y$ & $G_{2}(5)$ & $5^{6}$ & 9 & \\
\hline$T h$ & $2^{5} \cdot L_{5}(2)$ & $2^{15}$ & 9 & \\
\hline$F i_{23}$ & $S_{8}(2)$ & $2^{18}$ & 9 & $(6)$ \\
\hline$C o_{1}$ & $2_{+}^{1+8} \cdot O_{8}^{+}(2)$ & $2^{21}$ & 9 & \\
\hline$J_{4}$ & $2^{11}: M_{24}$ & $2^{21}$ & 27 & \\
\hline$F i_{24}^{\prime}$ & $3^{7} \cdot O_{7}(3)$ & $3^{16}$ & 9 & \\
\hline$B$ & $2 \cdot^{2} E_{6}(2) .2$ & $2^{41}$ & 12 & \\
\hline$M$ & $2 . B$ & $2^{46}$ & 36 & \\
\hline
\end{tabular}

TABLE 1. Upper bounds on the minimal length of special solvable conjugate factorzations of simple sporadic groups and the Tits group

\section{REFERENCES}

[1] M. Abért. Symmetric groups as products of abelian subgroups. Bull. London Math. Soc. 34 (2002), no. 4, 451-456. 
[2] J. H. Conway; R. T. Curtis; S. P. Norton; R. A. Parker; R. A. Wilson. Atlas of finite groups. Maximal subgroups and ordinary characters for simple groups. With computational assistance from J. G. Thackray. Oxford University Press, Eynsham, 1985.

[3] L. Babai; N. Nikolov; L. Pyber. Product growth and mixing in finite groups. Proceedings of the Nineteenth Annual ACM-SIAM Symposium on Discrete Algorithms, 248-257, ACM, New York, 2008.

[4] W. Bosma; J. Cannon; C. Playoust. The Magma algebra system. I. The user language. Computational algebra and number theory (London, 1993). J. Symbolic Comput. 24 (1997), no. 3-4, 235-265.

[5] T. Breuer. CTblLib, - GAP's Character Table Library package, version 1.2.1, (2012), http://www.math.rwth-aachen.de/ ${ }^{\sim}$ Thomas.Breuer/ctbllib

[6] T. Breuer; J. Müller. GAP file tst/mferctbl.gap, a compiled database of character tables of endomorphism rings of multiplicity-free permutation modules of the sporadic simple groups and their cyclic and bicyclic extensions.

[7] T. Breuer; K. Lux. The multiplicity-free permutation characters of the sporadic simple groups and their automorphism groups. Comm. Algebra 24 (1996), no. 7, 2293-2316.

[8] J. Cannon; M. Garonzi; D. Levy; A. Maróti; I. Simion. Groups equal to a product of three conjugates subgroups, to appear in Israel J. Math.

[9] R. W. Carter. Nilpotent self-normalizing subgroups of soluble groups. Math. Z. 75 (1960/1961) 136-139.

[10] R. W. Carter. Finite groups of Lie type. Conjugacy classes and complex characters. Reprint of the 1985 original. Wiley Classics Library. A Wiley-Interscience Publication. John Wiley \& Sons, Ltd., Chichester, 1993.

[11] J. D. Dixon; B. Mortimer. Permutation groups. Graduate Texts in Mathematics, 163. Springer-Verlag, New York, 1996.

[12] K. Doerk; T. Hawkes. Finite soluble groups. de Gruyter Expositions in Mathematics, 4. Walter de Gruyter \& Co., Berlin, 1992.

[13] D. Easdown; C. E. Praeger. On minimal faithful permutation representations of finite groups. Bull. Austral. Math. Soc. 38 (1988), no. 2, 207-220.

[14] E. I. Khukhro; P. Shumyatsky. Nonsoluble and non- $p$-soluble length of finite groups. Israel J. Math. 207 (2015), no. 2, 507-525.

[15] The GAP Group, GAP - Groups, Algorithms, and Programming, Version 4.6.5; 2013. (http://www.gap-system.org)

[16] M. Garonzi; D. Levy. Factorizing a finite group into conjugates of a subgroup. J. Algebra 418 (2014), 129-141.

[17] M. Garonzi; D. Levy; A. Maróti; I. Simion. Minimal length factorizations of finite simple groups of Lie type by unipotent Sylow subgroups, To appear in the Journal of Group Theory.

[18] M. Garonzi, D. Levy, A. Maróti; I. Simion. Primitive permutation groups as products of point stabilizers, arXiv:1508.05659.

[19] D. F. Holt; B. Eick; E. A. O'Brien. Handbook of computational group theory. Discrete Mathematics and its Applications (Boca Raton). Chapman \& Hall/CRC, Boca Raton, FL, 2005.

[20] B. Huppert. Endliche Gruppen. I. Die Grundlehren der Mathematischen Wissenschaften, Band 134 Springer-Verlag, Berlin-New York 1967.

[21] L. G. Kovács; C. E. Praeger. On minimal faithful permutation representations of finite groups. Bull. Austral. Math. Soc. 62 (2000), no. 2, 311-317.

[22] H. Kurzweil; B. Stellmacher. The theory of finite groups. An introduction. Universitext. Springer-Verlag, New York, 2004.

[23] M. W. Liebeck; N. Nikolov; A. Shalev. A conjecture on product decompositions in simple groups. Groups Geom. Dyn. 4 (2010), no. 4, 799-812.

[24] M. W. Liebeck; N. Nikolov; A. Shalev. Product decompositions in finite simple groups. Bull. Lond. Math. Soc. 44 (2012), no. 3, 469-472.

[25] M. W. Liebeck; L. Pyber. Finite linear groups and bounded generation. Duke Math. J. 107 (2001), no. 1, 159-171.

[26] L. Pyber; E. Szabó. Growth in linear groups. Thin groups and superstrong approximation, 253-268, Math. Sci. Res. Inst. Publ. 61, Cambridge Univ. Press, Cambridge, 2014.

[27] J. S. Rose. A course on group theory. Cambridge University Press, Cambridge-New YorkMelbourne, 1978. 
[28] A. Smolensky. Unitriangular factorization of twisted Chevalley groups. Internat. J. Algebra Comput. 23 (2013), no. 6, 1497-1502.

[29] N. A. Vavilov; A. V. Smolensky; B. Sury. Unitriangular factorizations of Chevalley groups. Zap. Nauchn. Sem. S.-Peterburg. Otdel. Mat. Inst. Steklov. (POMI) 388 (2011), Voprosy Teorii Predstavlenii Algebr i Grupp. 21, 17-47, 309-310; translation in J. Math. Sci. (N. Y.) 183 (2012), no. 5, 584-599

(Martino Garonzi) Departamento de Matematica, Universidade de Brasília, Campus Universitário Darcy Ribeiro, Brasília - DF 70910-900, Brasil

E-mail address: mgaronzi@gmail.com

(Dan Levy) The School of Computer Sciences, The Academic College of Tel-AvivYaffo, 2 Rabenu Yeruham St., Tel-Aviv 61083, Israel

E-mail address: danlevy@mta.ac.il

(Attila Maróti) Alfréd Rényi Institute of Mathematics, Reáltanoda utca 13-15, H1053, Budapest, Hungary

E-mail address: maroti.attila@renyi.mta.hu

(Iulian I. Simion) Department of Mathematics, University of Padova, Via Trieste 63, 35121 Padova, Italy

E-mail address: iulian.simion@math.unipd.it 